\title{
Les interventions de l'enseignant au travers de l'outil numérique : quels usages pour une EPS de qualité ? Illustration en natation de vitesse
}

\author{
Cyril Albertini
}

Cyril Albertini. Groupe EPIC, AEEPS \& Lycée Fesch, Académie de Corse, Ajaccio, France

Résumé. Notre intervention présente les résultats d'une recherche-action conduite avec le groupe EPIC en natation de vitesse. Nous proposons un dispositif de différenciation pédagogique fondé sur un " tableur numérique ", outil à la fois diagnostique, formatif et évaluatif, qui vise à optimiser la relation amplitude / fréquence afin d'améliorer la vitesse de chaque élève. Ancrée sur une analyse multi factorielle d'une part et les grandes statistiques qui s'en dégagent, nous proposons, grâce à l'outil numérique, des Zones Individualisées de Performance (ZIP) raisonnables, adaptées et équitables pour chacun. Pour autant nous engageons une réflexion sur l'usage et les limites de l'outil numérique dans le cadre de l'enseignement en EPS.

Mots-clés. Indicateurs de compétence, Tableur numérique, TICE

\section{Introduction}

Dès l'entrée dans le métier, de par le format des concours de recrutement, les jeunes enseignants d’EPS sont initiés aux usages du numérique et plus particulièrement de la tablette. Les enseignants d'EPS plus expérimentés sont également impactés par ce « raz-de-marée numérique » dans laquelle cette technologie peut être considérée comme un véritable « artefact numérique » (Mevel, 2017) source de développement chez les élèves comme pour les enseignants. L'usage du numérique s'impose jusqu'à tendre « vers une EPS $2.0 »$ (Morieux, 2014).

Mais, si « enseigner et apprendre avec le numérique » (Karsenti \& Bugmann, 2017) peut présenter de réels intérêts pour les enseignants, comme pour les élèves, il s'agit de « trouver un juste équilibre » pour éviter certains écueils. Régulièrement utilisé au sein de la leçon d’EPS, le numérique peut faire débat. Est-ce « une ressource, un gadget, voire une source d'aliénation ou d'addiction »? (Andrieu, 2015).

En nous appuyant sur notre «tableur ZIP»(Albertini, 2017), notre objectif est d'optimiser les interventions de l'enseignant pour aiguiller au mieux nos élèves vers des apprentissages qui soient raisonnables, adaptés et équitables pour chacun. S'il s'agit de pour prendre en compte et suivre les performances de chacun des élèves, nous interrogeons les avantages et limites de l'utilisation de cet outil sur la notation et l'évaluation par compétence. Nous questionnons également la place de la mixité et les modalités de mises en situation d'évaluation les plus favorables à la réussite de tous les élèves.

\section{Méthodologie}

Menée au sein du groupe EPIC (Evaluation Par Indicateur de Compétence - Roosli, Gibon, \& Rossi, 2015), cette rechercheaction s'appuie sur une situation de référence de $25 \mathrm{~m}$ sprint. Le tableur ZIP (Zone Individualisée de Performance) que nous proposons présente une partie relative à l'évaluation diagnostique et l'autre à l'évaluation finale.

Lors des évaluations diagnostiques et finales, après avoir composé les séries selon l'ordre alphabétique, le recueil de données consiste à saisir le temps et le nombre de coups de bras réalisés (observation élève) pour effectuer le $25 \mathrm{~m}$ sprint. Reposant sur une analyse multifactorielle (temps, nombre de coups de bras, performance initiale, thème de travail prescrit, sexe) et au regard des grandes tendances statistiques des 167 élèves de sixième (63 filles et 104 garçons) durant quatre années, et à la lumière d'indicateurs de compétence, l'enseignant d'EPS comme l'élève peuvent 
Les interventions de l'enseignant au travers de l'outil numérique : quels usages pour u...

immédiatement bénéficier de plusieurs informations.

A l'issue de l'évaluation diagnostique le tableur ZIP permet de prédire : aiguillage de chaque élève vers un thème de travail personnalisé (amplitude vs fréquence), mais aussi affichage d'un temps cible à atteindre en fin de séquence (figure 1). A l'issue de l'évaluation finale, il permet de: (1) prendre en compte l'affichage des paramètres de nage de chaque élève (amplitude, fréquence, indice de nage), du record fille-garçon et d'une proposition de note sur 20 ; (2) suivre ce que chaque élève produit (affichage d'un indicateur progrès - pourcentage de gain par rapport à sa performance initiale) ainsi que du niveau d'atteinte de sa ZIP en liaison avec un code couleur (ZIP « atteinte et au-delà » liée à la couleur or, « atteinte » liée à l’argent, « presque atteinte » en bronze et « non atteinte » en rouge).

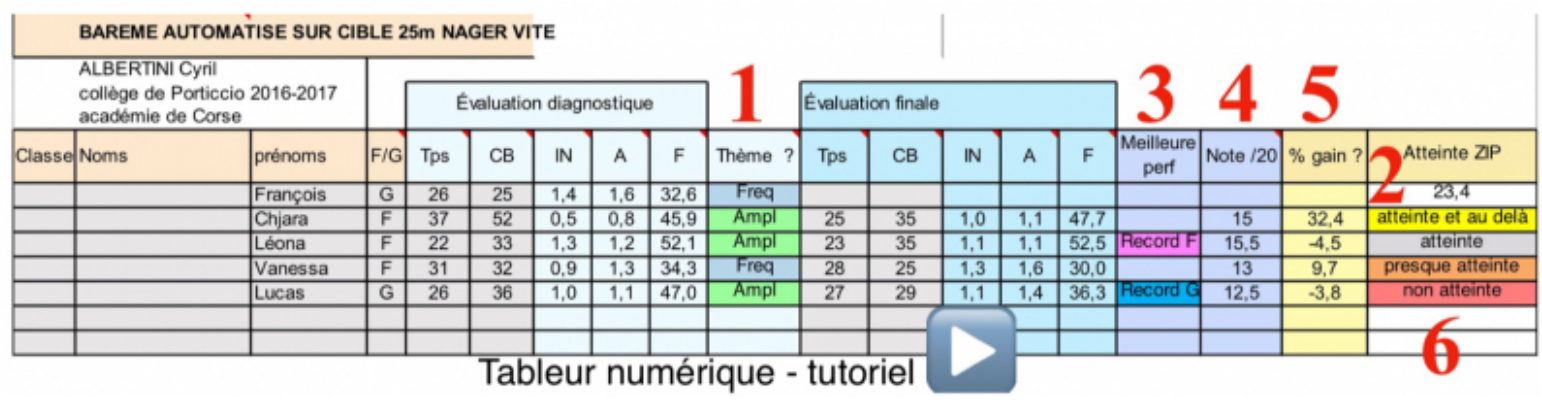

Figure 1 - Illustration du tableur ZIP

Avec un effectif de 50 élèves, nous avons testé les performances dans une autre modalité que l'ordre alphabétique : la modalité en opposition directe dans la «finale des champions » ou «finale espoirs » en fonction des niveaux de performance. Pour l'enseignant, l'intérêt premier de l'outil numérique, notamment la tablette, serait de combiner un encombrement réduit, une forte capacité de calcul et de stockage (datas, photos, vidéos) et un compagnon tout-terrain pour l'enseignant d'EPS en particulier (pour un usage en plein air ou encore sur le bord d'un bassin).

En répondant à la demande institutionnelle de « construire et utiliser des outils permettant l'évaluation des besoins, des progrès et du degré d'acquisition des compétences » (p.5 - Référentiel compétences professionnelles, 2015), nous souhaitons à la fois répondre au « comment ce monde de plus en plus en plus technologique peut-il être plus humain ?» (Blanquer, 2017) tout en tentant de « rendre l'évaluation certificative formatrice » (Meard \& Grandchamp, 2018). Ainsi, en s'appuyant sur des indicateurs de compétence qui lui permettent d'être plus précis et sur l'outil numérique qui permet un gain de temps tout en limitant les erreurs de calcul, la plus-value pour l'enseignant serait aussi de le rendre plus juste et plus disponible pour renforcer l'acte d'enseigner qui, selon nous, reste par-dessus tout un ensemble d'interactions profondément humaines (gestion de l'état de stress, des émotions, de l'engagement ... de chacun ou plus largement de la sécurité, du respect des règles ou du climat de classe). Le gain de temps peut aussi permettre à l'enseignant de faire, par moment, « un pas de côté » afin de porter son regard sous un nouvel angle, avec plus de recul sur la conduite de ses élèves en étant affranchi de ses feuilles, de ses tableaux ou de sa tablette numérique. Il s'agit de ne pas devenir un "enseignant addict" de l'outil numérique et d'accepter de s'en libérer, de façon « raisonnée » pour se centrer sur la façon dont l'élève mobilise ses ressources pour accomplir la tâche.

Pour l'élève, l'outil numérique peut aussi présenter une plus-value dans la mesure où il peut être vecteur d'apprentissages sociaux et méthodologiques, notamment au travers du rôle d'observateur, voire de conseiller.

Le recueil d'informations (nombre de coup de bras) et la manipulation de paramètres relatifs à l'action (amplitude, fréquence et indice de nage) est propice à la compréhension et, plus largement à l'acquisition de compétences méthodologiques et sociales. Au niveau de l'engagement des élèves, les informations fournies en direct par le tableur 
Actes de la 11ème Biennale de l'ARIS : Former des citoyens physiquement éduqués. Axe 2 - Vers une éducation physique de qualité, 10.25518/sepaps20.300

numérique (l'aiguillage, le contrat personnalisé, l'immédiateté de la note, l'atteinte de sa ZIP) sont de nature à déclencher / entretenir un comportement positif et volontaire.

\section{Résultats et discussion}

Plusieurs points interpellent notre réflexion. Tout d'abord, on peut constater qu'en modalités « ordre alphabétique », plus de $80 \%$ des élèves $(81,3 \% \mathrm{~F}, 82,1 \% \mathrm{G})$ progressent. Cette progression varie de 5 à $25 \%$ par rapport à la performance initiale. Ainsi, ce sont $80 \%$ des élèves qui sont aiguillés vers le thème de travail amplitude, alors que $20 \%$ sont orientés vers la fréquence. Par contre, le groupe fréquence affiche les marges de progression les plus importantes.

Par ailleurs, en modalité «finale des champions », 32\% des élèves nagent encore plus vite (dont plus de la moitié sont des filles). Ces progrès sont probablement liés au fait que les élèves seraient plus activé.e.s par un but d'accomplissement de type autrui-approche (Elliot et al., 2011).

Si $32 \%$ des élèves ont réussi à nager plus vite en confrontation directe, on constate que $20 \%$ ont réalisé le même temps et que $48 \%$ ont nagé moins vite. Ainsi, nous pensons que l'enseignant qui ne propose qu'une seule modalité d'évaluation (réaliser la meilleure performance mesurée à partir d'une série fondée uniquement sur la modalité « ordre alphabétique » ou «finale des champions ») ne se donne pas les moyens d'accéder à la totalité du potentiel de tous ses élèves.

De façon globale, la marge d'amélioration moyenne est de 9,9\% (jusqu'à 19,4\%).

Quels sont les élèves qui sont capables de telles améliorations ? En se référant à la figure 2, on peut constater que les élèves qui se sont améliorés en situation d'opposition (32\%), sont ceux qui avaient une ZIP « atteinte » (20\%), « presque atteinte » $(4 \%)$ et «non atteinte » $(8 \%)$. Aucun.e élève n'avait une ZIP « atteinte et au-delà ».

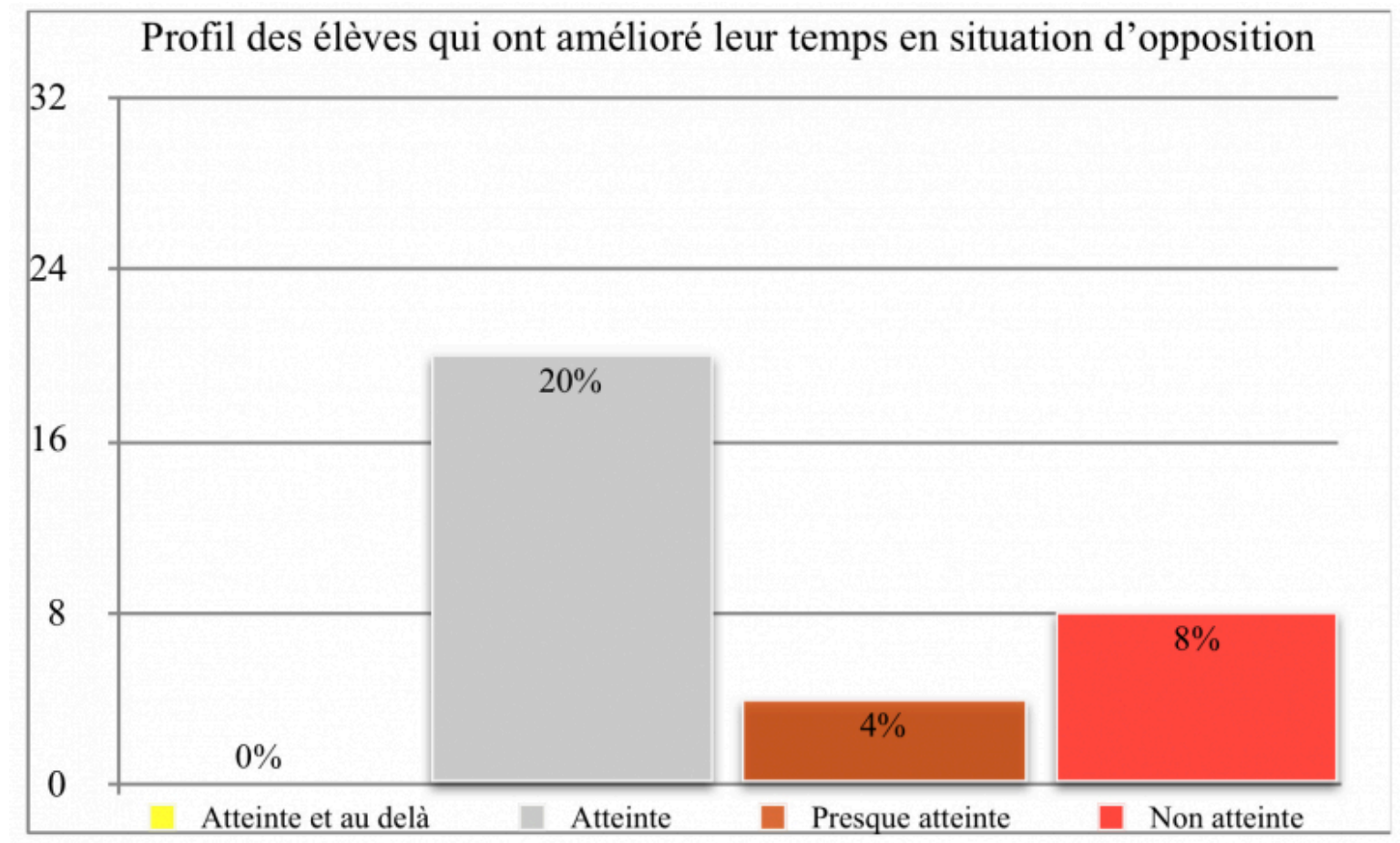

Figure 2 - Profil des élèves qui ont amélioré leur temps en situation d’opposition 
Les interventions de l'enseignant au travers de l'outil numérique : quels usages pour u...

Pour s'améliorer autant, ces élèves ont dû jouer sur certains paramètres, mais lesquels ? L'amplitude et/ou la fréquence et/ou le départ et/ou la coulée et/ou la reprise de nage et/ou ...? Est-ce une mobilisation différente des ressources énergétiques, bio-mécaniques ... voire psycho-affectives ? Cette deuxième course, en situation d'opposition a pu permettre à certains de s'améliorer et cela peut simplement s'expliquer par le fait d'avoir eu une deuxième chance pour certain,e,s (mauvais départ, perte de matériel, ...) ou par le fait d'être activés par l'opposition (but d'accomplissement de type autrui-approche - Mascret, 2018).

La figure 3 nous montre que pour la majorité des élèves qui améliorent leur temps, on trouve 16\% de filles et 12\% de garçons pour un total de $28 \%$ sur $32 \%$ et que cela résulte de l'amélioration de la fréquence (d'ailleurs dans des proportions de 5,3 à 63,5\%). De plus, dans ce contexte d'opposition, on peut remarquer qu'une amélioration importante de la fréquence est souvent couplée avec une perte en amplitude, ce qui au final, ne se traduit pas nécessairement par une amélioration importante du temps.

Seuls $4 \%$ des élèves ont réussi à améliorer leur temps en améliorant uniquement le paramètre amplitude de nage.

À nouveau, la figure 3 nous permet d'identifier que les filles et les garçons sont concernés de la même façon au regard de la gestion des paramètres (amplitude, fréquence) de gestion de la vitesse. Ici, l'outil numérique nous permet de rapidement vérifier la dimension équitable de notre enseignement. Ainsi, on peut constater que, dans cette modalité d'opposition, plus de la moitié des élèves qui s'améliorent sont des filles (66,6\%). Pour 80\% d'entre elles, principalement à partir d'une amélioration de la fréquence et pour $20 \%$ d'entre elles, à partir d'une amélioration de l'amplitude.

Cela dit, nous n'oublions pas que dans cette modalité d'opposition certains élèves ont régressé. Si 32\% des élèves ont réussi à nager plus vite, on constate que $20 \%$ ont réalisé le même temps et que $48 \%$ ont nagé moins vite. Cela peut s'expliquer par l'effet fatigue pour certains ou par le stress engendré par l'opposition pour d'autres (but d'accomplissement de type autrui-évitement).

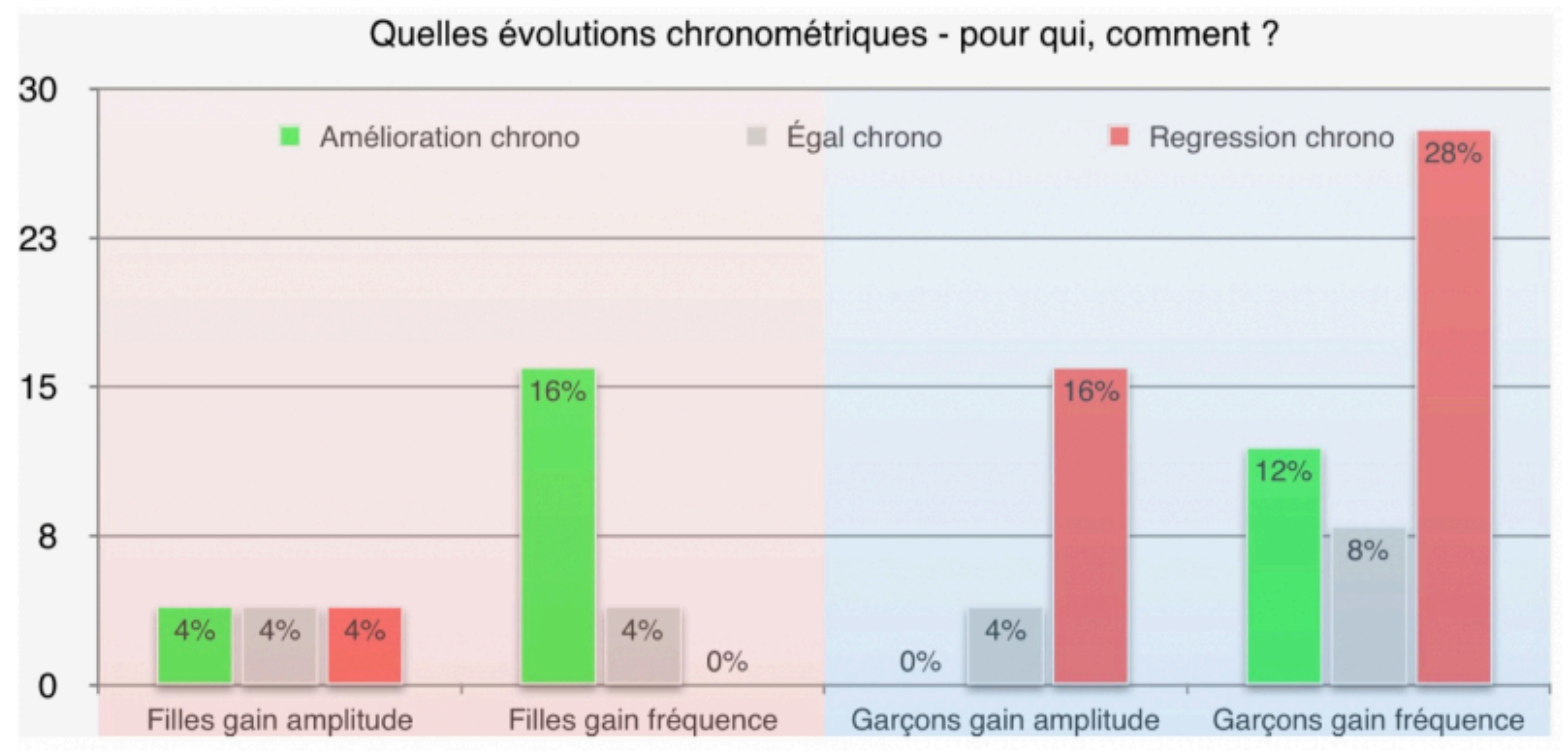

Figure 3 - Quelles évolution chronométriques? Pour qui, comment? 
Actes de la 11ème Biennale de l'ARIS : Former des citoyens physiquement éduqués. Axe 2 - Vers une éducation physique de qualité, 10.25518/sepaps20.300

\section{Conclusions}

Pour permettre à chacun.e d'exprimer tout son potentiel, il est indispensable de proposer différentes modalités d'évaluation (Albertini, 2019). Si le numérique apporte une réelle plus-value, en permettant à l'enseignant d'être plus rapide et plus précis, il peut montrer ses limites car l'activité humaine n'est pas totalement numérisable. L'outil informatique, perçu comme un artefact, doit donc avoir une place réfléchie dans les pratiques enseignantes. Ce n'est ni une loi, ni une fin en soi et il est bon de savoir s'en détacher de temps en temps. Si dans l'acte d'enseigner, «l'humain » (la relation, le ressenti, l'intuition, ....) disparait, nous pouvons passer à côté de certaines choses.

\section{Bibliographie}

Andrieu, B. (2015). Les usages du numérique "ressource ou gadget », Conférence sur les nouvelles technologies en EPS, Rouen. Disponible sur : http://eps.spip.ac-rouen.fr/spip.php?article1650

Albertini C. (2017). Des indicateurs de compétence pour aider l'enseignant à déterminer des Zones Individualisées de Performance. Revue Enseigner l'EPS, 273, 14-16

Albertini C. (2019). L'outil numérique, intérêt et limites en EPS. Illustration avec le tableur ZIP en natation de vitesse. Dossier AEEPS nº4. Enseigner l'EPS avec le numérique : pour quels apprentissages ?, 77-81

Blanquer, J.-M. (2017). Lettre aux professeurs et aux personnels de l'Education Nationale.

Elliot A. J., Murayama K., \& Pekrun R. (2011). A 3 x 2 achievement goal model. Journal of educational psychology, 103/ $3,632-648$.

Karsenti, T., \& Bugmann, J. (Eds.) 2017. Enseigner et apprendre avec le numérique. Montréal : Presses de l’Université de Montréal.

Mascret, N. (2018). Vivre une expérience authentique en EPS. Conférence du CRIEPS, Créteil.

Meard, J., \& Grandchamp, A. (2018). Les différentes formes d'évaluation. In M. Travert \& O. Rey (Eds.): L'engagement de l'élève en EPS. Dossier EPS n85 (pp. 171-177). Paris : Revue EP.S.

Mevel, C. (2017). La tablette en classe : un outil motivationnel sous conditions ! Les acteurs du savoir.

Morieux, M. (2014). Vers une EPS 2.0 ? Revue EPS, 360, 38-39.

Roosli, W., Gibon, J., \& Rossi, D. (2015). L'évaluation par indicateur de compétences, un outil au service de la réussite de tous. Revue Enseigner I'EPS, 268, 2-6.

PDF automatiquement généré le 2021-11-17 22:51:02

Url de l'article : https://popups.uliege.be/sepaps20/index.php?id=300

Publié par ULiège Library en Open Access et distribué suivant les termes et les conditions de la licence CC-BY (https://creativecommons.org/licenses/by/4.0/deed.fr) 